\title{
VELLEIO PATERCOLO, AUGUSTO E L'OMBRA LUNGA DI TIBERIO
}

\begin{abstract}
Summary: Velleius Paterculus' judgement of Augustus and of the early years of Tiberius' reign was deeply influenced by his standpoint on Tiberius and the circumstances of his succession. But it is clear that the historian could lay aside his prejudice while writing the biography of Augustus and estimate the most important persons (Marcellus, Agrippina, Caius and Iulius Caesar) who played an important role at the death of Augustus and were rivals of Tiberius relatively unbiased.
\end{abstract}

Key words: Octavianus Augustus, Tiberius, Agrippa, Gaius Caesar, Lucius Caesar, successio

La celebre monografia di Italo Lana su Velleio Patercolo (Velleio Patercolo o della propaganda, Torino 1952) intendeva mostrare come la Storia Romana di Velleio più che un'opera storiografica fosse uno scritto propagandistico a favore di Tiberio inteso a promuovere il nuovo corso politico inaugurato dal successore di Augusto soprattutto attraverso la promozione degli homines novi. ${ }^{1}$ Pochi anni dopo Emilio Gabba, in un noto contributo ristampato in Esercito e società (1973), comparso originariamente nel 1962 sulle pagine di Critica Storica, ${ }^{2}$ aveva messo in luce come il filo conduttore del primo libro della Storia di Velleio andasse rintracciato nel problema dell'estensione della civitas romana agli italici, orientando così l'opera stessa di Velleio, un italico di Aeclanum, in una prospettiva italica. ${ }^{3}$ Velleio insomma era dotato di una precisa prospettiva storica nel narrare la storia di Roma fino al I secolo d.C.: come è noto egli prendeva le mosse dalla guerra di Troia - anche se purtroppo l'opera in due libri è fortemente mutila nel primo libro essendo caduti gli avvenimenti compresi tra il co-

\footnotetext{
${ }^{1}$ Per le critiche alla tesi del Lana cfr. almeno HeLlegouARC'H, J.: Les buts de l'œuvre historique de Velleius Paterculus. Latomus 23 (1964) 669-684.

${ }^{2}$ GABBA, E.: Italia e Roma nella «Storia» di Velleio Patercolo. In ID.: Esercito e società nella tarda repubblica romana. Firenze 1973, 347-360 (= CS 1 [1962] 1-9).

${ }^{3}$ Così anche MAZZARINO, S.: Il pensiero storico classico. Vol. I-II. Bari 1965-1966, 433-434.
} 
siddetto ratto delle sabine e la terza guerra macedonica - per giungere sino al 30 d.C. l'anno del consolato di Marco Vinicio a cui l'opera è dedicata. In un così vasto arco di tempo erano ovviamente compresi anche gli eventi relativi al principato augusteo che tuttavia non possono essere integralmente ricompresi né nella lettura del Lana né in quella del Gabba. Vero è che il termine "propaganda" appare quanto mai appropriato in riferimento alla narrazione di Velleio sul principato di Tiberio; altrettanto si può dire del principato di Augusto dal momento che un primo dato che balza all'occhio in modo evidente nel leggere la Caesarian Augustan Narrative (2. 41-93) è l'indubbia tendenza di Velleio favorevole prima a Cesare e poi ad Augusto (la benevolenza verso Cesare appare quasi una premessa indispensabile in relazione al giovane Ottaviano poi Augusto). Qualche esempio relativo ad Ottaviano-Augusto appare di palmare evidenza: Ottaviano è il vero erede di Cesare ed è la vittima delle insidie di Antonio sin da subito, non appena viene nominato erede di Cesare (2. 61. 3-5); Lepido è eletto fraudolentemente (furto) Pontefice Massimo al posto di Ottaviano (2. 63. 1); la responsabilità delle proscrizioni è attribuita ad Antonio e a Lepido «con l'opposto parere di Cesare» (2. 66. 1-2); Velleio, in almeno un paio di passi (2. 61. 1-3 e 89. 3-4), richiama così da vicino il testo delle Res gestae di Augusto che è impossibile non pensare che lo utilizzasse come fonte. ${ }^{5}$

Come il ritratto velleiano di Tiberio, senz'altro deformato in melius, ma anche caratterizzato da una sincera e profonda ammirazione, ${ }^{6}$ costituisce pur sempre un correttivo all'immagine altrettanto deformata in peius che troviamo in Tacito, Suetonio e Cassio Dione, così l'immagine di Ottaviano-Augusto, sebbene anch'essa profondamente deformata da Velleio in melius, appare dettata dalla sua profonda ammirazione e dalla sincera adesione al nuovo regime inaugurato da Augusto. ${ }^{7}$ Tuttavia, recentemente, un interessante contributo di U. Schmitzer ${ }^{8}$ ha ripreso in esame le pagine velleiane relative alla clades variana di Teutoburgo del 9 d.C. ha messo in luce alcune difficoltà e taluni imbarazzi mostrati dal nostro storico nell'affrontare certi nodi particolarmente critici del principato augusteo. A me sembra che questa impostazione possa offrire nuovi spunti di riflessione che intendo qui sviluppare ed arricchire ulteriormente.

\footnotetext{
${ }^{4}$ Woodman, A. J.: Velleius Paterculus. The Caesarian and Augustan narrative (2.41-93). Cambridge 1983. Per la mendacità di Velleio basti SYME, R.: Mendacity in Velleius. AJPh 99 (1978) 45-63.

${ }^{5}$ Cfr. soprattutto HellegouARC'H, J. - JODRY, CL.: Les Res Gestae et l'Historia Romana de Velleius Paterculus. Latomus 39 (1980) 803-816; cfr. anche 2. 96. 3.

${ }^{6}$ Che Velleio non nasconde a se stesso, conscio dei possibili rimproveri che gli potrebbero essere rivolti, cfr. 2. 116. 5: Horum virorum mentioni si quis quaesisse me dicet locum, fatentem arguet: neque enim iustus sine mendacio candor apud bonos crimini est.

${ }^{7}$ Per la communis opinio negli studi velleiani cfr. SCHMITZER, U.: Velleius Paterculus und das Interesse an der Geschichte im Zeitalter des Tiberius. Heidelberg 2000, 13-24.

${ }^{8}$ SCHMITZER, U.: Tatsachenbericht oder literarische Fiktion? Velleius Paterculus über die clades Variana. In LehmanN, G. A. - WIEGELs, R. (Hrsg.): Römische Präsenz und Herrschaft im Germanien der augusteischen Zeit. Der Fundplatz von Kalkriese im Kontext neuerer Forschungen und Ausgrabungsbefunde. Göttingen 2007, 399-417.
} 
Un primo dato che colpisce è che nei primi otto capitoli il riferimento cronologico sulla base del quale sono datati tutti gli avvenimenti è la conclusione della guerra di Troia, non la fondazione di Roma. Ciò che soprattutto sorprende è la mancata menzione di Enea - profugo da Troia e progenitore della stirpe Giulia - e il suo approdo sulle coste del Lazio. La fondazione dell'Urbe è senz'altro attribuita a Romolo e della saga troiana non è fatta parola, ma soprattutto il nome di Enea non compare mai in tutta l'opera di Velleio. Lascia alquanto perplessi poi che a 1. 6. 4 sia ricordata Elis$\mathrm{sa} /$ Didone come fondatrice di Cartagine «sessantacinque anni prima della fondazione di Roma» mentre del suo amante Enea non vi è la benché minima traccia. La distanza con Virgilio non potrebbe essere maggiore.

Tuttavia non so quanto questo silenzio riveli una polemica con Augusto. ${ }^{9}$ È innegabile infatti che Augusto pose particolare attenzione a presentare se stesso come nuovo fondatore di Roma,${ }^{10}$ ma rifutò il nome di Romolo. Forse è meglio pensare che Velleio avesse in mente Tiberio più che Augusto. Tiberio infatti era un Claudio, adottato da Augusto nella gens Iulia, e da Claudio si era sempre comportato - celebri sono le pagine di Tacito in cui si ricorda la durezza e la fierezza della gens Claudia ben impersonata da Tiberio ${ }^{11}$ - cercando, sia prima di assumere il governo dell'impero (si pensi allo sdegnato ritiro a Rodi) sia dopo averlo assunto (si pensi ai rapporti con Agrippina Maggiore), di tenere sempre le debite distanze dai Giuli che ai suoi occhi rappresentavano una minaccia sia per sé che per l'impero: Agrippina Maggiore e la sua discendenza - ma anche la moglie Giulia - erano molto probabilmente un ricorrente pensiero negativo per Tiberio e senz'altro un grave intralcio dal punto di vista politico. Velleio era ben consapevole del fatto che i rapporti tra Augusto e suo figlio adottivo nonché successore non sempre erano stati cordiali e pertanto si doveva regolare di conseguenza: non era opportuno riaprire vecchie ferite, né era necessario che la famiglia di Augusto oscurasse quella di Tiberio, e dunque un calcolato silenzio sulla propaganda augustea in riferimento alla gens Iulia - che poteva fare anche a meno della voce di Velleio nel trarre ulteriore alimento tra i contemporanei - non doveva danneggiare il nostro storico più di tanto. Egli infatti si guarda bene dal mettere in dubbio in alcun modo la legittimità della successione di Tiberio ad Augusto: il giovane Tiberio era per Velleio principe già nell'aspetto (2. 94. 2) e alla morte di Augusto «una sola fu, per così dire, la lotta della città: il senato e il popolo romano doverono opporsi a Cesare perché succedesse al posto del padre, mentre preferiva essere un cittadino pari agli altri piuttosto che avere il ruolo eminente di principe» $(2.124 .2)^{12}$.

\footnotetext{
${ }^{9}$ SCHMitZer: Velleius Paterculus (n. 7) 43-60.

${ }^{10}$ Basti ricordare a mo' d'esempio la celebre battuta contenuta in Suet. Aug. 28. 3: urbem neque pro maiestate imperii ornatam et inundationibus incendiisque obnoxiam excoluit adeo, ut iure sit gloraitus marmoream se relinquere, quam latericiam accepisset.

${ }^{11}$ Cfr. ad es. Tac. Ann. 1. 4. 3; Livio (2. 56. 79) definiva la gens Claudia superbissima et crudelissima in plebem Romanam; per la fama sinistra della gens cfr. anche Suet. Tib. 1-2. Per il conflitto tra Giuli e Claudi cfr. LEVICK, B.: Julians and Claudians. $G \& R 22$ (1975) 29-38.

${ }^{12} \mathrm{La}$ traduzione dei passi velleiani è di ElEfanTe, M. (ed.): Velleio Patercolo. I due libri al console Marco Vinicio. Napoli 1999.
} 
Di fatto il vero tramite agli occhi di Velleio per la successione di Tiberio fu Livia $^{13}$ alla quale il nostro storico dedica un passo molto elogiativo $(2.75 .3)^{14}$ relativo al tempo delle guerre civili. L'interesse del passo risiede nel fatto che Livia, nonostante sia ritratta con in braccio il figlioletto Tiberio in fuga insieme al marito Tiberio Claudio Nerone oppositore di Ottaviano, riceve un ampio elogio da Velleio: vero è che il nonno di Velleio aveva aiutato Tiberio Claudio Nerone e la sua famiglia a fuggire da Napoli, quando Tiberio, che a Perugia si era schierato con Lucio Antonio, dopo la sconfitta aveva provocato una ribellione di espropriati a Preneste e poi a Napoli ${ }^{15}$ e in seguito si era rifugiato in Sicilia presso Sesto Pompeo e ancora in Grecia presso Antonio ${ }^{16}$ ma la chiave per comprendere il passo è il matrimonio di Livia con Ottaviano nel 38 a.C., che le consentì di portare in dote al futuro imperatore Tiberio stesso. ${ }^{17}$ Molto probabilmente qui Velleio, che era ben consapevole del fatto che il rapporto tra Augusto e Tiberio non fosse sempre stato idilliaco proprio in relazione alla successione (Augusto gli preferì sempre qualcun altro: prima Marcello, poi Agrippa e infine Gaio e Lucio Cesari), sente il bisogno di legittimare Tiberio, mostrando come Ottaviano in virtù del suo matrimonio con Livia aveva avuto da subito la possibilità di nominare Tiberio come legittimo successore (e non lo fece). Anche in tal caso non si può escludere che il risalto dato qui a Livia - nient'affatto necessario in questo luogo della narrazione velleiana - celi un biasimo per come Augusto avesse condotto la lunga e tormentata vicenda della sua successione, sebbene anche in tal caso Velleio proceda con molta cautela.

Il discorso su Livia e Tiberio porta inevitabilmente con sé anche quello relativo agli oppositori di Ottaviano dal momento che, come s'è accennato, oltre al fugace ritratto di Tiberio Claudio Nerone ciò che colpisce in queste pagine di Velleio è l'atteggiamento verso Cassio, Bruto e Cicerone, vale a dire gli ultimi repubblicani.

A proposito di Cassio e Bruto Velleio afferma che (2. 69. 6) «non si potrebbero trovare uomini che o siano stati accompagnati da una fortuna più favorevole di quella

${ }^{13}$ Sul conto della quale peraltro le fonti ostili non mancarono di speculare imbastendo una presunta ostilità tra figlio e madre che non aveva ragione d'essere. GALIMBERTI, A.: Fazioni politiche e principesse imperiali (I-II sec. d.C.). CSA 7 (2009) 121-153, 122-132. Cfr. anche Velleio 2. 130. 4-5: Cuius temporis aegritudinem auxit amissa mater, eminentissima et per omnia deis quam hominibus similior foemina, cuius potentiam nemo sensit nisi aut levatione periculi aut accessione dignitatis.

${ }^{14}$ Livia, nobilissimi et fortissimi viri Drusi Claudiani filia, genere probitate forma Romanarum eminentissima, quam postea coniugem Augusti vidimus, quam transgressi ad deos sacerdotem ac filiam, tum fugiens mox futuri 〈viri〉 sui Caesaris arma, 〈mi ’nus bimum hunc Ti. Caesarem, vindicem Romani imperii futurumque eiusdem Caesaris filium, gestans sinu, per avia itinerum vitatis militum gladiis uno comitante, quo facilius occultaretur fuga, pervenit ad mare et cum viro Nerone pervecta in Siciliam est.

${ }^{15}$ Anche in tal caso Velleio ha cura di sottolineare la grandezza del padre di Tiberio, chiudendo poi telegraficamente la notizia con l'arrivo di Ottaviano e il suo decisivo intervento (2. 75. 1): Per eadem tempora exarserat in Campania bellum, quod professus eorum, qui perdiderant agros, patrocinium ciebat Ti. Claudius Nero praetorius et pontiiex, Ti. Caesaris pater, magni vir animi doctissimique et ingenii. Id quoque adventu Caesaris sepultum atque discussum est.

${ }^{16}$ Cfr. anche Tac. Ann. 5. 1; Suet. Tib. 4. 6; Dio 48. 15. 3; 44. 1.

${ }^{17}$ Per il matrimonio con Livia e le sue implicazioni politiche cfr. ora RoHR VIO, F.: Le nozze di Augusto tra azione politica e strategie propagandistiche. In LUCIANI, S.: Entre mots et marbre. Les metamorphoses d'Auguste. Bordeaux 2016, 53-66. Su Livia cfr. ora BRACCESI, L.: Livia. Roma 2016. 
di Bruto e Cassio o che, poi, come se l'avessero stancata, siano stati abbandonati da lei più in fretta» e, dopo la narrazione di Filippi, tributa loro il seguente riconoscimento (2. 72. 1-2): «Questa fu la fine che la fortuna volle dare al partito di Marco Bruto (M. Bruti partium). Egli aveva allora trentasette anni, ed era stato di animo puro fino al giorno che, per la temerarietà di un solo gesto, gli portò via ogni virtù. Cassio come generale, era stato superiore a Bruto, quanto questi lo era come uomo. Tra i due sarebbe stato preferibile avere come amico Bruto, come nemico sarebbe stato maggiormente temibile Cassio. Nell'uno ci fu maggiore forza, nell'altro maggiore valore; e se avessero vinto, quanto più utile è stato per lo Stato avere Cesare come principe invece di Antonio, altrettanto lo sarebbe stato avere Bruto piuttosto che Cassio»».

È dunque la fortuna ad abbandonare Bruto e Cassio ai quali, nonostante i loro gravi errori, va riconosciuto di avere intrapreso una guerra - quella contro i triumviri che poteva anche essere vinta conducendoli così a governare (in tal caso la preferenza di Velleio è chiaramente per Bruto). In realtà a me sembra che il giudizio molto moderato su Bruto e Cassio si spieghi alla luce del fatto che per Velleio il vero centro dell'attenzione, vale a dire il vero nemico, è Antonio il quale, se avesse avuto la meglio su Ottaviano, avrebbe rappresentato una sciagura per la res publica (quantum rei publicae interfuit Caesarem potius habere quam Antonium principem). Che in cima ai pensieri del nostro storico vi fosse Antonio mi sembra confermato e contrario dalla narrazione della battaglia di Filippi in cui il ruolo di Antonio, il vero artefice della vittoria sui cesaricidi, è del tutto oscurato a favore di una clamorosa falsificazione a favore di Ottaviano il quale - «benché fosse molto malato e fosse stato scongiurato dal suo medico Artorio di non rimanere nell'accampamento» - appare come l'unico generale a combattere per la parte cesariana e ad ottenere la vittoria. Nel clima di pacificazione voluto dall'ultimo Augusto (dopo il 2 a.C.) Bruto e Cassio potevano tutto sommato godere anche di una parola benevola, Antonio no.

Del resto sappiamo che Augusto aveva letto l'opera di Bruto su Catone al quale si era preso la briga di rispondere con i rescripta Bruto de Catone (la cui lettura pubblica, ormai vecchio, aveva affidato a Tiberio) ${ }^{18}$ nonché quella di Cremuzio Cordo che Tiberio si era guardato bene dal lasciare circolare liberamente e che aveva determinato la fine del suo autore nel 25 d.C. ${ }^{19}$

Vale la pena notare inoltre che mentre Augusto nelle Res gestae (1. 1), attribuisce alla dominatio di una factio l'oppressione della res publica lasciando contem-

${ }^{18}$ Suet. Aug. 85. 1: Multa varii generis prosa oratione composuit, ex quibus nonnulla in coetu familiarium velut in auditorio recitavit, sicut Rescripta Bruto de Catone, quae volumina cum iam senior ex magna parte legisset, fatigatus Tiberio tradidit perlegenda.

${ }^{19}$ Dio 57. 24. 2-3 «Cremuzio Cordo fu costretto a togliersi la vita di sua mano perché era entrato in contrasto con Seiano; del resto non fu possibile sottoporlo ad alcuna accusa rilevante (infatti era ormai alle porte della vecchiaia ed aveva vissuto in modo assolutamente irreprensibile), e perciò venne processato sulla base della sua opera storica, che egli aveva composto molto tempo prima sulle imprese di Augusto e che Augusto stesso aveva letto: le accuse erano quelle di aver elogiato Cassio e Bruto, di aver criticato il popolo e il senato e, infine, sebbene non avesse mai parlato negativamente né di Cesare né di Augusto, di non aver comunque mostrato un giusto rispetto nei loro riguardi». 
poraneamente intendere in modo molto sottile e ambiguo che la factio era sì quella di Antonio ma anche quella di cui parlava Cesare nel Bellum civile (vale a dire la factio rappresentata dagli optimates), ${ }^{20}$ Velleio (2. 61. 1) attribuisce diligentemente al solo Antonio l'oppressione della civitas trasferendo quasi di peso l'espressione delle Res gestae: ${ }^{21}$ il vero nemico di Velleio non è, come per Augusto, l'opposizione anticesariana e poi antoniana, bensì il solo Antonio, reo di avere messo a morte Cicerone.

Per Velleio infatti Antonio è soprattutto l'ispiratore e il responsabile unico delle proscrizioni $^{22}$ e dunque in primis della morte di Cicerone. Questi è peraltro uno degli eroi dell'opera di Velleio al quale dedica un capitolo dove si parla delle proscrizioni e della sua fine. Che Cicerone sia un personaggio di primo piano nel pensiero di Velleio $^{23}$ è rivelato innanzitutto dal fatto che l'arpinate appare sullo stesso piano di Ottaviano (2. 66. 2): «Nessuna azione in quel periodo fu così vergognosa quanto il fatto che o Cesare fosse costretto a proscrivere qualcuno o che da qualcuno fosse proscritto Cicerone. Per la scelleratezza di Antonio fu messa a tacere quella voce del popolo romano, senza che nessuno avesse tentato di difendere la vita di colui che pure per tanti anni aveva tutelato l'integrità dello stato e la salvezza privata dei cittadini»». Al di là della centralità di Cicerone e del falso prodotto da Velleio di cui si è già detto (Ottaviano non fu affatto estraneo alle proscrizioni), c'è un terzo aspetto che colpisce in questo passo: il fatto che Velleio non riesce a nascondere il suo dissenso per il fatto che neppure Ottaviano alzò un dito a favore di Cicerone: cum eius (scil. Ciceronis) salutem nemo defendisset. Se da un lato dunque Velleio sembra solennemente discolpare Ottaviano dalle proscrizioni, dall'altro non può fare a meno di lasciare intendere che lo stesso Ottaviano (forse proprio in quanto estraneo alle proscrizioni) avrebbe potuto fare di più in favore Cicerone. Certo, subito dopo Velleio prorompe nella più feroce invettiva di tutta la sua opera contro Antonio, addossandogli tutta la

${ }^{20}$ BC 1. 22. 5: ut se et populum Romanum factione paucorum oppressum in libertatem vindicaret.

${ }^{21} R G$ 1. 1: Annos undeviginti natus exercitum privato consilio et privata impensa comparaui, per quem rem publicam a dominatione factionis oppressam in libertatem uindicaui; Vell. 2. 61. 1: Torpebat oppressa dominatione Antoni civitas ... cum C. Caesar undevicesimum annum ingressus ... priuato consilio maiorem senatu pro re publica animum habuit. Per il commento del passo del $R G$ cfr. ora SCHEID, J. (ed.): Res gestae Divi Augusti. Hauts Faits du divin Auguste. Paris 2007, 27-28; per il passo di Velleio e la sua terminologia cfr. KOBER, M.: Die politische Anfänge Oktavians in der Darstellung des Velleius und dessen Verhältnis zur historiographischen Tradition, Würzburg 2000.

${ }^{22} \mathrm{Mi}$ domando se, parlando del proscritto Tiberio Claudio Nerone in fuga verso la Sicilia (cfr. supra), Velleio in ultima analisi non imputasse la sua fuga presso Sesto Pompeo proprio ad Antonio in quanto responsabile delle proscrizioni. Velleio infatti a 2.72. 4 scrive che presso Sesto Pompeo «si dirigevano in massa dall'accampamento di Bruto, dall'Italia e da altre parti del mondo i proscritti che la fortuna aveva sottratto all'imminente pericolo».

${ }^{23}$ SCHMITZER: Velleius Paterculus (n. 7) 184-189. Non escluderei che alla base ci potessero essere anche motivazioni personali; Velleio infatti (2.15) ricorda più volte la fedeltà verso Roma del suo antenato Minato Magio, mostrata in occasione di gravi pericoli esterni fin dal tempo delle guerre annibaliche. Egli è uno di quei boni homines novi la cui promozione sociale era stata senz'altro favorita da Cicerone: proprio grazie a uomini come loro, capaci di far sentire la loro voce anche contro gli esponenti della classe senatoriale, si sarebbe fondato il consensus omnium bonorum vagheggiato da Cicerone nella Pro Sestio; si veda soprattutto LEPORE, E.: Il princeps ciceroniano e gli ideali politici della tarda repubblica. Napoli 1954, 9-19. 
colpa della morte di Cicerone,$^{24}$ ma ciò non vale a cancellare quel che ha detto poco prima. Di più: c'è un destino comune che lega Bruto, Cassio e Cicerone: sono tutti vittime delle proscrizioni e, seppure in maniera diversa, rappresentano gli ultimi repubblicani. Questo spazio positivo concesso agli oppositori di Ottaviano serve infine a Velleio per accomunarli a Tiberio Claudio Nerone, legittimando così il padre di Tiberio. È altrettanto chiaro però che anche questa legittimazione deve essere letta in riferimento alla successione voluta da Augusto che, nella sua complessa evoluzione, aveva creato momenti di tensione tra il principe e Tiberio. Come è noto, Augusto si era risolto ad adottare Tiberio nel 4 d.C. soltanto alla fine di una lunga e faticosa vicenda. Il principe infatti da subito aveva cercato un successore all'interno della famiglia, puntando prima su M. Marcello, primo marito di Giulia Maggiore (figlia di Augusto e Scribonia, sua seconda moglie), morto improvvisamente nel 23 a.C.; poi su Agrippa, morto anch'egli prematuramente nel 12 a.C. e quindi sui nipoti, adottati come figli, Gaio Cesare e Lucio Cesare, figli di Giulia e e di Agrippa: entrambi però erano disgraziatamente morti giovani (Lucio nel 2 d.C. e Gaio nel 4 d.C.); solo da ultimo Augusto scelse Tiberio, al quale era stata fatta balenare già in precedenza la successione, prima attraverso il matrimonio con Giulia, rimasta vedova di Agrippa nel 12 a.C. e ora al suo terzo matrimonio, poi con il conferimento della tribunicia potestas nel 6 a.C. per cinque anni.

Tuttavia, di fronte alla "designazione" di Lucio e Gaio nel 6 a.C., Tiberio si era sdegnosamente ritirato a Rodi, per poi fare ritorno a Roma qualche anno dopo, nel 2 d.C. Soltanto dopo la morte di Gaio Cesare nel 4 d.C. il cammino verso la successione appariva spianato: oltre alla morte dei nipoti di Augusto infatti anche la madre Giulia (nonché moglie di Tiberio) era caduta in disgrazia nel 2 a.C. (seguita pochi anni dopo, nell'8 d.C., dalla figlia Giulia Minore), mentre nel 4 d.C. Tiberio, insieme ad Agrippa Postumo (l'ultimo figlio di Giulia e Agrippa, che soffriva però di tare mentali) era stato adottato come figlio da Augusto ed aveva ricevuto nuovamente la tribunicia potestas per altri dieci anni (che nel 13 gli fu rinnovata). Sennonché di lì a poco, nel 6 d.C., Agrippa fu esiliato dapprima a Sorrento e poi, definitivamente a Planasia (tra il 7 e l's d. C.) e Tiberio non ebbe più avversari. Nel 13 ricevette l'imperium consulare, che lo rendeva "collega" di Augusto nonché l'imperium proconsulare (il comando sulle province e sugli eserciti, il cosiddetto imperium maius, la cui natura è però discussa) al pari di quello di Augusto. Nel 14, alla morte di Augusto, Tiberio divenne imperatore: il primum facinus novi principatus (Tac. Ann. 1. 6. 1), consumato per ordine di Augusto, fu l'uccisione di Agrippa Postumo.

${ }^{24}$ 2. 66. 3-4: Nihil tamen egisti, M. Antoni (cogit enim excedere propositi formam operis erumpens animo ac pectore indignatio) nihil, inquam, egisti mercedem caelestissimi oris et clarissimi capitis abscisi numerando auctoramentoque funebri ad conservatoris quondam rei publicae tantique consulis inritando necem. Rapuisti tu M. Ciceroni lucem sollicitam et aetatem senilem et vitam miseriorem te principe quam sub te triumviro mortem, famam vero gloriamque factorum atque dictorum adeo non abstulisti, ut auxeris. 
Queste vicende sono condensate da Velleio (secondo la tecnica del compendium adottata nella Storia caratterizzata da brevitas e festinatio $)^{25}$ al capitolo 93 del secondo libro: «Cinquanta anni fa, morì, ancora molto giovane, Marco Marcello, figlio di Ottavia, sorella di Augusto. Si riteneva generalmente che, se qualche disgrazia fosse accaduta a Cesare, egli sarebbe dovuto succedergli nel potere, pur essendo, tuttavia, diffusa la convinzione che ciò non potesse toccargli in tutta tranquillità a causa di Marco Agrippa. Egli, in qualità di edile, aveva dato uno splendido spettacolo, ed era in vero, come dicono, fornito di nobili virtù, di buon carattere e ingegno, all'altezza della fortuna per la quale era allevato. Dopo la sua morte, Agrippa, che con il pretesto di importanti incarichi imperiali era partito per l'Asia ma, come corre voce, a causa di contrasti con Marcello si era sottratto alla presente circostanza, rientrò e sposò la figlia di Cesare, Giulia, che già era stata moglie di Marcello, donna di non felice fecondità, né per se né per lo Stato».

Il giudizio su Agrippa, nel complesso positivo, conserva tuttavia un margine di ambiguità: per Velleio Agrippa è innanzitutto un'ombra per la successione di Marcello, il candidato voluto da subito da Augusto, che non amava particolarmente e a causa del quale si sarebbe allontanato nel 23 a.C. andandosene a Lesbo. A ben guardare è lo stesso atteggiamento assunto da Tiberio quando decise di andarsene a Rodi nel 6 a.C. per non ostacolare con la sua presenza i figli di Agrippa e di Giulia (Gaio e Lucio Cesare). ${ }^{26}$ Sorge pertanto il dubbio che Velleio qui abbia forzato un po' la mano, attribuendo ad Agrippa ciò che in realtà aveva compiuto Tiberio. Si badi però che il confronto tra Agrippa e Tiberio, implicito e a distanza, ma molto chiaro nelle pagine della Storia Romana, è nettamente favorevole a Tiberio: mentre il "ritiro" di Agrippa è dettato dall'invidia e dai contrasti con Marcello, il ritiro vero e proprio di Tiberio è senz'altro un meraviglioso gesto d'affetto verso i figli di Agrippa (2. 99. 2: mira quadam et incredibili atque inenarrabili pietate).

Certo è che, dopo Marcello, il candidato di Augusto era proprio il suo fidatissimo collaboratore e generale Agrippa: le parole di Velleio sembrano dunque stridere con la designazione voluta da Augusto in seconda istanza; si aggiunga, e questo mi sembra ancora più importante, che l'aspra critica a Giulia (feminam neque sibi neque rei publicae felicis uteri) non consiste solo nell'accusa di sovversione politica - aspetto questo senz'altro molto filoaugusteo - bensì anche nel fatto di aver generato Gaio e Lucio Cesari che furono la causa dell'autoesilio di Tiberio a Rodi. È chiaro insomma che Velleio legge tutta la successione voluta da Augusto in funzione di Tiberio per cui la "rivalutazione" - per così dire - di Agrippa avviene soltanto nel momento in cui si spalancano le porte alla successione di Tiberio (cfr. 2. 96. 1): «In seguito la morte di Agrippa - che aveva nobilitato le sue origini di uomo nuovo con numerose imprese, ed era giunto fino al punto che era suocero di Nerone e che il divino Augusto aveva adottato i suoi figli, che gli erano nipoti, con i nomi di Gaio e di Lucio - avvicinò

\footnotetext{
${ }^{25}$ Sono termini impiegati da Velleio stesso per definire la sua opera: 1. 16. $1 ; 2.38 .1 ; 66.3 ; 96.3$ (forma propositi operis); 2. 52. 3; 89. 1; 103.4 (festinatio); 2. 55. 1; 86. 1; 99.4 (transcursus, celeritas).

${ }^{26}$ Cfr. LEVICK, B.: Tiberius' retirement to Rhodes in 6 B.C. Latomus 31(1972) 779-813.
} 
di più Nerone a Cesare. Sua figlia Giulia, infatti, giá moglie di Agrippa, sposò Nerone». ${ }^{27}$ L'assenza assoluta di qualsivolgia giudizio su Giulia, precedentemente attacata in modo feroce, e ora semplicemente sposa di Tiberio, nonché il silenzio sul doloroso divorzio di Tiberio da Vipsania (la figlia di Agrippa!), lasciano chiaramente intendere che qui a scrivere è il Velleio cantore ufficiale della propaganda augusteo-tiberiana. Resta il fatto che poco oltre Agrippa viene paragonato a Seiano: ${ }^{28}$ come il potente prefetto del pretorio di Tiberio, Agrippa resta agli occhi di Velleio un collaboratore; certo il miglior collaboratore possibile nonché il più fidato (2. 79. 1: parendi, sed uni, scientissimus) ma pur sempre un collaboratore, non il successore designato. Mi sembra quantomai significativo che "l'epitaffio" di Agrippa in Velleio in realtà sia rivolto ad esaltare Tiberio: ${ }^{29}$ il merito di Agrippa insomma sarebbe per Velleio quello di aver agevolato con la sua morte la successione di Tiberio; persino il comando della guerra Pannonica, che segue nello stesso capitolo la morte di Agrippa, non è tanto l'occasione per esaltare le doti di generale di Agrippa quanto quelle di Tiberio, che grazie alla vittoria su Pannoni e poi sui Dalmati aveva ottenuto l'onore dell'ovatio ed aveva soprattutto liberato l'Italia da una minaccia.

Per quanto riguarda Tiberio è indubbio dunque che il suo regno per Velleio comincia con la morte di Agrippa nel 12 a.C., quando il figlio di Livia assunse la tribunicia potestas ${ }^{30}$ (cosa che, formalmente, appare esattissima); ecco perché in corrispondenza del ritiro di Tiberio Rodi nel 6 a.C. (protrattosi per sette anni, vale a dire fino all' 1 d.C.), Velleio afferma che i promagistrati (proconsoli o legati) si recavano là a rendere omaggio a Tiberio, pur essendo egli soltanto un privato $e$, in virtù della sua straordinaria autorevolezza, abbasavano i fasci «riconoscendo che la sua vita privata era più meritevole di onore del loro potere politico» (2.99.4).

Sulla base di questa premessa si comprende anche perché le parole di Velleio su Gaio Cesare (figlio di Agrippa e di Giulia) suonino quantomeno "diplomatiche" (2. 101. 1): il suo comportamento in Siria, dove era stato inviato da Augusto per incontrare il re dei Parti Fraatace nell' 1 d.C., è ritenuto da Velleio «così contraddit-

${ }^{27}$ Mors deinde Agrippae, qui novitatem suam multis rebus nobilitaverat atque in hoc perduxerat ut et Neronis esset socer, cuiusque liberos nepotes suos divus Augustus praepositis Gai ac Lucii nominibus adoptaverat, admovit propius Neronem Caesari; quippe filia eius Iulia, quae fuerat Agrippae nupta, Neroni nupsit.

28 2. 127. 1-3: Raro eminentes viri non magnis adiutoribus ad gubernandam fortunam suam usi sunt, ut duo Scipiones duobus Laeliis, quos per omnia aequaverunt sibi, ut divus Augustus M. Agrippa et proxime ab eo Statilio Tauro, quibus novitas familiae haut obstitit quominus ad multiplicis consulatus triumphosque et complura eveherentur sacerdotia. Etenim magna negotia magnis adiutoribus egent interestque rei publicae quod usu necessariurn est, dignitate eminere utilitatemque auctoritate muniri. Sub his exemplis Ti. Caesar Seianum Aelium, principe equestris ordinis patre natum, materno vero genere clarissimas veteresque et insignes honoribus complexum familias, habentem consularis fratres, consobrinos, avunculum, ipsum vero laboris ac fidei capacissimum.

${ }^{29}$ 2. 96. 1: Mors deinde Agrippae, qui novitatem suam multis rebus nobilitaverat atque in hoc perduxerat, ut et Neronis esset socer, cuiusque liberos nepotes suos divus Augustus praepositis Gai ac Lucii nominibus adoptaverat, admovit propius Neronem Caesari: quippe filia Iulia eius, quae fuerat Agrippae nupta, Neroni nupsit. Subinde bellum Pannonicum, quod inchoatum ab Agrippa, Marco Vinicio, avo tuo consule, magnum atroxque et perquam vicinum imminebat Italiae, per Neronem gestum est.

${ }^{30}$ Tac. Ann. 1. 56. 2; Dio 54. 31. 2; LEVICK: Tiberius' retirement (n. 26) 782. 
torio che non mancherebbero validi argomenti a chi volesse lodarlo, né sufficienti a chi volesse biasimarlo»e anche in tal caso, ad occupare la scena ancora una volta non è Gaio bensì Tiberio il quale, pur essendo dapprima stato onorato da Gaio, viene poi fatto oggetto di un comportamento "contradditorio". Velleio allude molto probabilmente al fatto che Tiberio si era recato a Samo per rendere omaggio a Gaio (che in quel momento deteneva un imperium maius) e questi lo aveva ricevuto molto freddamente; la testimonianza di Velleio peraltro qui è molto preziosa poiché è egli stesso a dichiarare che all'epoca dei fatti era stato testimone oculare quando era tribuno militare $(\S 3) .^{31}$

Certo, rimaneva anche la candidatura di Agrippa Postumo - violentemente attaccato da Velleio come era naturale (2.112.7) - ma, mai come in questo caso Augusto e Tiberio furono concordi: quello che Tacito definisce come il primum facinus novi principatus attribuendolo a Tiberio era stato in realtà un ordine di Augusto che Tiberio non aveva esitato ad eseguire all'indomani della morte di Augusto stesso.

La strategia di Velleio a questo punto appare sufficientemente scoperta: la costruzione del personaggio (perché di ciò si tratta) di Tiberio prima del suo avvento al principato in rapporto agli altri attori - Augusto e la sua discendenza - prevede un calcolato impiego di accenni e silenzi al fine di non tacere da una parte i meriti di Tiberio stesso e dall'altra non oscurare quelli di Augusto. Questi poteva essere criticato senz'altro (cosa che puntualmente avviene) ma non più di tanto sia perché Velleio, come s'è detto, aveva aderito profondamente e sinceramente al principato augusteo ritenendolo una costruzione ampiamente positiva, sia perché ciò si sarebbe rivelato controproducente anche per Tiberio, il successore di Augusto che con il suo governo andava a perfezionare l'opera del predecessore.

A questo proposito appare esemplare l'atteggiamento di Velleio in merito alla clades Variana del 9 d.C. come ha messo bene in luce il recente contributo di Ulrich Schmitzer a cui si è fatto cenno. Non solo Augusto non compare nel resoconto della disfatta di Teutoburgo - neppure come responsabile della scelta di Varo, che certo non appare dotato di sufficienti virtù morali e militari - ma anche Tiberio è assente, nonostante avesse avuto molteplici rapporti con Varo e fosse con lui imparentato, ${ }^{32}$ di più: il personaggio di Varo sembra costruito come l'antitipo di Tiberio, il quale aveva talento e virtù militari da vendere; Varo infine, in quanto generale tragicamente sconfitto era comunque indifendibile. Per Velleio tutta la responsabilità del disastro ricade dunque inevitabilmente sulle spalle di Varo, un indolente, corrotto, incapace di condurre un esercito e di capire il nemico, ${ }^{33}$ come se la sconfitta dovesse

${ }^{31}$ Su Tiberio e Gaio cfr. ora PISTEllato, A.: Gaius Caesar, or the Ideal Non-princeps: A Tiberian Issue. Arctos 47 (2013) 199-217.

${ }^{32}$ Imparentato con Augusto (e dunque anche con Tiberio) attraverso il matrimonio con una parente di Ottavia (sorella di Augusto), era stato console con Tiberio nel 13 a.C.

${ }^{33}$ Precisa è l'accusa contro Varo di essersi fatto trarre in inganno dai Germani (2.118. 1): at illi [scil. Germani], quod nisi expertus vix credat, in summa feritate versutissimi natumque mendacio genus, simulantes fictas litium series et nunc provocantes alter alterum in iurgia, nunc agentes gratias quod ea Romana iustitia finiret feritasque sua novitate incognitae disciplinae mitesceret et solita armis decerni iure terminarentur, in summam socordiam perduxere Quintilium, usque eo ut se praetorem urbanum in foro ius dicere, non in mediis Germaniae finibus exercitui praeesse crederet. 
essere esorcizzata imputandola unicamente ai suoi limiti e ai suoi difetti. Certo alla disgrazia avevano contribuito anche la perfidia del nemico e la cattiva sorte, tuttavia vale la pena notare, come ha fatto recentemente Giusto Traina, la stretta analogia con il caso della sconfitta di Crasso a Carre del 53 a.C. (il paragone del resto si trova già in Velleio): ${ }^{34}$ Crasso, analogamente a Varo, appare l'unico responsabile dell'insuccesso, soprattutto a motivo della sua insipienza. Questo eccesso di personalismo (nella sconfitta) in Crasso (e poi in Varo), segna peraltro una svolta nella concezione della sconfitta a Roma: «Anche in questo caso (scil. quello di Varo), come per Crasso, il disonore viene elaborato a distanza di tempo, e un disastro pubblico veniva trasformato in disastro privato, dove la sorte si era accanita sul comandante per la sua incapacità di gestire la crisi militare». ${ }^{35}$

Velleio appare anche in tal caso in perfetta sintonia con i suoi tempi, se intendiamo questa espressione come sinonimo di adesione a quella che doveva essere la versione della clades Variana che probabilmente circolava sotto Tiberio. ${ }^{36}$

Questo atteggiamento infine sintetizza meglio di ogni altro la posizione del nostro storico nei riguardi di Augusto da una parte e di Tiberio dall'altra che ho cercato qui di individuare. Mi sembra che per Velleio il doveroso e sincero omaggio ad Augusto non doveva oscurare la figura di Tiberio, al quale andava altrettanta gratitudine. ${ }^{37}$ Questo equilibrio, talvolta difficile, stante soprattutto il passato di Augusto quando era ancora Ottaviano e i suoi rapporti con Tiberio nella lunga e tormentata vicenda della successione, era l'obiettivo che Velleio intendeva raggiungere nella sua opera. Alla luce di questo obiettivo mi pare possano essere spiegati gli episodi che ho proposto: la falsificazione delle vicende triumvirali a favore di Ottaviano; il silenzio su Enea e la gens Iulia tanto propagandato da Augusto e oscurato da Velleio; l'esaltazione di Livia in funzione della piena legittimazione della successione di Tiberio al quale Augusto aveva anteposto sempre i discendenti diretti della famiglia Giulia; il "recupero" di Cicerone ${ }^{38}$ (con annessa critica ad Ottaviano), ma anche di Bruto e Cassio, a cospetto di Antonio, dettato significativamente dalla svolta del 2 a.C. quando Augusto affrontando la grande crisi del suo principato dovette allargare il suo consenso alle file veterorepubblicane; l'ambiguo giudizio su Agrippa e il diplomatico giudizio su Gaio Cesare; il duro giudizio su Varo.

${ }^{34}$ 2. 119. 1: Ordinem atrocissimae calamitatis, qua nulla post Crassi in Parthum damnum in externis gentibus gravior Romanis fuit iustis voluminibus ut alii, ita nos conabimur exponere: nunc summa deflenda est. L'analogia è estensibile anche al fatto che entrambi i comandanti erano morti in battaglia.

${ }^{35}$ TrainA, G.: Le sconfitte dei Romani. Aevum 84 (2010) 177-185, 184.

${ }^{36}$ Augusto, come è noto, nelle Res Gestae preferì sorvolare sulla disfatta puntando sui successi in Germania (l'estensione del limes fino all'Elba). Per la costituzione della provincia di Germania sotto Augusto cfr. ECK, W.: Augustus und die Grossprovinz Germanien. Kölner Jahrbuch 37 (2004) 11-22; ZECCHINI, G.: La politica di Roma in Germania da Cesare agli Antonini. Aevum 84 (2010) 187-198. Tiberio

${ }^{37}$ Si ricordi che Velleio era stato pretore nel 14 d.C. e comandante della cavalleria sul Reno con

${ }^{38}$ Plutarco (Comp. Dem. et Cic. 50. 3. 1) ricorda che Augusto nel De vita sua aveva valorizzato la figura di Cicerone scrivendo che «il giovane Cesare aveva avuto bisogno dell'aiuto di Cicerone». 
È dunque in questa - volontaria - oscillazione tra le ragioni di Augusto e quelle di Tiberio che credo sia racchiuso il significato più profondo delle scelte storiografiche di Velleio sul primo principato.

\author{
Alessandro Galimberti \\ Dipartimento di Storia, Archelogia e Storia dell'Arte \\ Università Cattolica del Sacro Cuore \\ Sede di Milano \\ Largo A. Gemelli 1 \\ 20123 Milano \\ Italy
}

